\title{
EDITORIAL
}

\section{Disease monitoring in chronic obstructive pulmonary disease: is there a role for biomarkers?}

\author{
P.L.M.L. Wielders, P.N.R. Dekhuijzen
}

\begin{abstract}
Biological or biochemical markers (so-called biomarkers) are of potential interest for clinical application to monitor chronic diseases. Although biomarkers are frequently used in toxicology and oncology, their application in monitoring nonmalignant pulmonary disease has been confined to basic research. With the appearance of new noninvasive and less invasive markers it is tempting to speculate on their potential role in patients with chronic obstructive pulmonary disease (COPD).
\end{abstract}

\section{COPD: current perspective}

COPD is known to have major morbidity and mortality [1]. Since the presence and severity of the airflow limitation are associated with the prognosis with respect to survival, it is not only important to identify risk factors for the development of COPD, but also to prevent worsening of the disease in an early stage, in order to alter the natural history, especially in those who will have a rapid decline in pulmonary function. In epidemiological studies, risk factors for a rapid decline are marked bronchial hyperresponsiveness, high number of pack-years, low value of postbronchodilator forced expiratory volume in one second (FEV1) and low reversibility [2]. An individual estimation of the risk for presence or absence of a (future) rapid decline, however, is difficult to make. Sensitive clinical tools to establish the diagnosis at an earlier stage and to monitor the activity of the disease are not yet available. The present possibilities for an objective follow-up of patients with COPD consist of measurements of lung function at regular intervals [2]. However, lung function data only partially reflect the ongoing pathological process and are, therefore, too insensitive to monitor the actual state of the disease.

\section{Biomarkers?}

The question now arises whether biomarkers will be helpful in the identification of these individual risk factors. Biomarkers include markers of exposure to external influences, markers of susceptibility to that specific

Dept of Pulmonary Diseases, University Hospital Nijmegen, Nijmegen, The Netherlands.

Correspondence: P.L.M.L. Wielders, Dept of Pulmonary Diseases, University Hospital Nijmegen, P.O. Box 9101, 6500 HB Nijmegen, The Netherlands. disease and markers of pathophysiological changes related to the disease. Biomarkers of exposure are important if environmental or biological factors are studied, e.g. in case of occupational exposure or follow-up during smoking cessation programmes. They are of less importance in monitoring disease activity. Ideally, markers also provide insight into the susceptibility to the disease, are well correlated with the actual state of the pathological process and reflect the clinical severity of the disease. For applicability in respiratory disease, these biomarkers should have specific properties. They should accurately reflect the changes in disease activity in the target organ, in this case the lung. This requires a high sensitivity and specificity. They should be reproducible in steady state, easy to obtain, preferably in a noninvasive fashion, thereby facilitating repeated measurements. They should also reflect the changes due to therapy and, finally, be of prognostic significance. A biomarker of susceptibility (besides deficiency of $\alpha_{1-}$ proteinase inhibitor) may reveal why some smokers are at risk of developing COPD and others are not. A biomarker of effect (other than lung function data) would be valuable, e.g. in assessing a rationale for therapeutic intervention. Clearly, in COPD there is a need for more sensitive markers to follow the course of the disease and especially to determine which patients are susceptible for a rapid decline. To determine which biomarkers are potentially suitable parameters to be used in COPD, clues may be found in the evaluation of the pathological process.

\section{COPD: inflammation and oxidative stress}

Inflammatory processes in the bronchi, bronchioli and alveoli and their possible influence on oxidative stress are thought to play a crucial role in the development of COPD [3-5]. There is an active ongoing inflammatory process in both the central airways and the parenchyma. The inflammatory processes also influence the balance between oxidants and antioxidants. An increased number of neutrophils and macrophages, and an increase in their activity, accounts for an increased production of reactive oxygen species (ROS). ROS can damage cellular lipid membranes and molecules such as proteins and deoxyribonucleic acid (DNA). Intracellular and extracellular anti-oxidative defence mechanisms protect the lung against ROS, in which the glutathione redox cycle plays an important role.

Accordingly, biomarkers in COPD should reflect both 
the inflammatory nature and oxidative stress, express their relationship to the intensity of the pathological process and predict the individual outcome. If a biomarker, or a panel of biomarkers, with those properties is available, the method of acquisition still remains to be determined.

\section{Current biomarkers}

Clearly, biomarkers obtained by bronchoscopy are not suitable for the clinical follow-up of these patients. However, these invasive measures reflect the ongoing pathological process and, thus, should provide guidance towards development and application of less invasive biomarkers. Repeated observations in bronchoalveolar lavage (BAL) and biopsies show the significance of neutrophil activity in COPD. Increased number of neutrophils in BAL and their increased activity [3] is correlated with air flow limitation.

In contrast to the invasive procedures, examination of (induced) sputum offers several opportunities in COPD. Cellular markers, markers of activation and anti-oxidative parameters can be measured, although potential changes in biomarkers due to the technique of sputum induction itself (e.g. neutrophilia?) may be underestimated. Recently, Keatings et al. [6] showed increased levels of interleukin (IL)-8 (recruitment and activation of neutrophils) in patients with COPD, compared with asthmatics, healthy smokers and nonsmokers. In addition, myeloperoxidase and human neutrophil lipocalin (HNL), both markers of neutrophil activity, were significantly higher in COPD patients compared to asthmatics and healthy controls [7]. These results suggest that markers of neutrophil activation may be more specific for COPD than for asthma. These findings are in accordance with findings in BAL, suggesting that induced sputum and BAL reflect similar changes, at least in this respect. In the same study [6], tumour necrosis factor$\alpha(\mathrm{TNF}-\alpha)$, which acts as an activator of adhesion molecules on endothelial cells and thereby influences the chemotaxis of eosinophils, was elevated in both COPD and asthma. This supports the concept that the eosinophil, the most prominent cell in asthma, is not without significance in COPD. Probably it is not only the number of eosinophils, but also their activated state that accounts for this finding. Evidence for this hypothesis was provided in another study by the same authors [7], demonstrating that eosinophilic activation markers were raised in induced sputum, both of patients with COPD and asthma, whereas the total numbers of eosinophils per millilitre were similar [7]. BAL data however, do not confirm these observations. Data on anti-oxidative parameters (e.g. glutathione) in induced sputum are not yet available, but preliminary data from our laboratory indicate an increased concentration of total glutathione in induced sputum of patients with smoking-related COPD in comparison with healthy controls [8]. In BAL, an inverse correlation was found between the predicted value of FEV1 and the concentration of total glutathione [3]. The significance of these parameters in induced sputum in relation to the site of disease activity remains to be determined.

In the past, several inflammatory parameters have been studied in blood and urine, but the correlation with the pathological process in the target organ, i.e. the lung, is weak, except for the markers of eosinophilic activation. Eosinophilia is associated with a more rapid decline in lung function in newly diagnosed chronic bronchitis [9]. Monitoring anti-oxidant activity in plasma, however, is more likely to reflect changes in the pathological process. Both acute and chronic smoking decrease the Trolox equivalent anti-oxidant capacity (TEAC) of plasma [10]. TEAC values of patients who were admitted to the hospital with exacerbated COPD, were lower than those with stable disease and returned to similar values at discharge. In spite of that, elevated levels of thiobarbituric acid-reactive substances (TBARS) in plasma, as a marker of lipid peroxidation, were found both in stable and exacerbated COPD [10]. Total glutathione in plasma is another measure of anti-oxidant capacity, but its significance is not clear. Whether systemic changes mirror the changes in lung tissue has not yet been fully elucidated. Acute exposure to inhaled smoke decreases glutathione, whereas in chronic exposure increased levels of glutathione have been found in the epithelial lining fluid [11]. This is in contrast to findings in plasma, in which no difference was observed between nonsmokers and smokers [11].

Measurement in exhaled air is a noninvasive procedure, which provides biomarkers of use in clinical applications. Whereas online measurement of exhaled nitric oxide, which reflects the enhanced production in airway mucosal inflammation, is increased in asthma, this could not be demonstrated in a preliminary study in COPD [12]. However, in patients with COPD a higher concentration of hydrogen peroxide (mainly produced by activated macrophages and neutrophils) in breath condensate was found in comparison with healthy nonsmokers, with a further increase in exacerbated COPD [13]. Although this finding is not specific for COPD [14], this marker may reflect the intensity of inflammation and oxidative stress in COPD. Elevated levels of TBARS in breath condensate, as markers of lipid peroxidation, have been demonstrated in asthma [15], but not yet in COPD. The influence of various factors on the formation of condensate can be avoided with online measurement of exhaled gases. Exhaled shortchain alkanes (pentane, ethane, ethene) are considered to be markers of oxidant-induced lipid peroxidation. Indeed, online detected levels of ethane were raised in smokers compared to nonsmokers [16]. In a study [17] on the effect of supplemental anti-oxidant vitamin on ethane levels, a close inverse correlation was found between the decline in ethane output and the presupplemental FEV1. The authors concluded that the supplement of anti-oxidant vitamins attenuates the smoking-related lipid peroxidation and that preserved lung function is reflected in ethane output.

\section{From basics to clinical practice?}

It is clear that inflammation and oxidative stress are closely related to the development and course of COPD. Recent developments of biomarkers in research, may provide clinical tools which provide earlier and more sensitive monitoring than lung function data. Whether 
these markers will be applicable in clinical practice largely depends on the simplicity of measurement, sensitivity, specificity, predictive value and accuracy.

Online measurement of exhaled gases or measurement in breath condensate certainly has this potential with respect to the simplicity of sample collection, but its role in disease monitoring remains to be investigated. Markers derived from blood and urine have similar properties, but may lack the accurate reflection of changes in the target organ. Sputum induction, on the other hand, at least mirrors changes in the central airways, but performance of this test is rather time-consuming and strenuous for the patient. Before the definite introduction of several biomarkers to the clinical tool box, considerable effort has to be taken to understand some of the conflicting data. Therefore, future research should be focused on the validation of the different biomarkers, i.e. demonstration of their close correlation to the pathological process. In addition, search for new accurate biomarkers should be continued, if a real change in the natural history of COPD is pursued. Finally, analogous to the correlation of the births of babies and the appearance of the stork in spring, caution should be taken not to correlate all kinds of biomarkers, without any logical link in the concept of the pathological process.

\section{References}

1. Burrows B, Bloom JW, Traver GA, Cline MG. The course and prognosis of different forms of chronic airways obstruction in a sample from the general population. N Engl J Med 1987; 317: 1309-1314.

2. Siafakas NM, Vermeire P, Pride NB, et al. Optimal assessment and management of chronic obstructive pulmonary disease. Eur Respir J 1995; 8: 1398-1420.

3. Linden M, Rasmussen JB, Piitulainen E, et al. Airway inflammation in smokers with nonobstructive and obstructive chronic bronchitis. Am Rev Respir Dis 1993; 148: 1226-1232.

4. Jeffery PK. Morphology of the airway wall in asthma and in chronic obstructive pulmonary disease. Am Rev Respir Dis 1991; 143: 1152-1158.
5. Bast A, Haenen GRMM, Doelman CJA. Oxidants and antioxidants: state of the art. Am J Med 1991; 91 (Suppl. $3 \mathrm{C})$ : $2 \mathrm{~S}-13 \mathrm{~S}$.

6. Keatings VM, Collins PD, Scott DM, Barnes PJ. Differences in interleukin- 8 and tumor necrosis factor- $\alpha$ in induced sputum from patients with COPD or asthma. Am J Respir Crit Care Med 1996; 153: 530-534.

7. Keatings VM, Barnes PJ. Granulocyte activation markers in induced sputum: comparison between chronic obstructive pulmonary disease, asthma, and normal subjects. Am J Respir Crit Care Med 1997; 155: 449-453.

8. Wielders PLML, Dekhuijzen PNR, Ruytenbeek K, et al. Total glutathione is increased in induced sputum of COPD patients. Am J Respir Crit Care Med 1997; 155: A188.

9. Lebowitz MD, Postma DS, Burrows B. Adverse effects of eosinophilia and smoking on the natural history of newly diagnosed chronic bronchitis. Chest 1995; 108: 55-61.

10. Rahman I, Morrison D, Donaldson K, MacNee W. Systemic oxidative stress in asthma, COPD, and smokers. Am J Respir Crit Care Med 1996; 154: 1055-1060.

11. Cantin AM, North SL, Hubbard RC, Crystal RG. Normal alveolar epithelial lining fluid contains high levels of glutathione. J Appl Physiol 1987; 63: 152-157.

12. Rutgers SR, Postma DS, van der Mark TW, Koeter GH. Nitric oxide in exhaled air in COPD. Eur Respir J 1996; 9: Suppl. 23, 13s.

13. Dekhuijzen PNR, Aben KKH, Dekker I, et al. Increased exhalation of hydrogen peroxide in patients with stable and unstable COPD. Am J Respir Crit Care Med 1996; 154: 813-816.

14. Dohlman AW, Black HR, Royall JA. Expired breath hydrogen peroxide is a marker of acute airway inflammation in pediatric patients with asthma. Am Rev Respir Dis 1993; 148: 955-960.

15. Antczak A, Nowak D, Shariati B, et al. Increased hydrogen peroxide and thiobarbituric acid-reactive products in expired breath condensate of asthmatic patients. Eur Respir J 1997; 10: 1235-1241.

16. Habib MP, Clements NC, Garewal HS. Cigarette smoking and ethane exhalation in humans. Am J Respir Crit Care Med 1995; 151: 1368-1372.

17. Do BKQ, Garewal HS, Clements NC, Peng YM, Habib MP. Exhaled ethane and antioxidant vitamin supplements in active smokers. Chest 1996; 110: 159-164. 